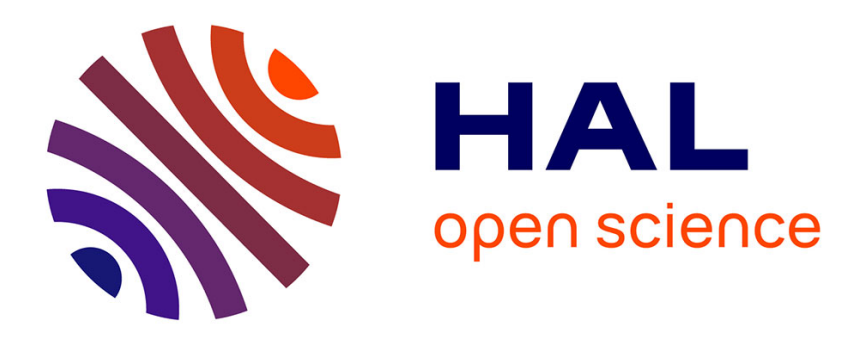

\title{
The virtual sub-channel protocol for satellite link communications
}

Guy Pujolle, O. Spaniol

\section{To cite this version:}

Guy Pujolle, O. Spaniol. The virtual sub-channel protocol for satellite link communications. [Research Report] RR-0038, INRIA. 1980. inria-00076523

\section{HAL Id: inria-00076523 \\ https://hal.inria.fr/inria-00076523}

Submitted on 24 May 2006

HAL is a multi-disciplinary open access archive for the deposit and dissemination of scientific research documents, whether they are published or not. The documents may come from teaching and research institutions in France or abroad, or from public or private research centers.
L'archive ouverte pluridisciplinaire HAL, est destinée au dépôt et à la diffusion de documents scientifiques de niveau recherche, publiés ou non, émanant des établissements d'enseignement et de recherche français ou étrangers, des laboratoires publics ou privés. 
THE VIRTUAL SUBCHANNEL PROTOCOL

FOR SATELLITE LINK COMMUNICATIONS

by

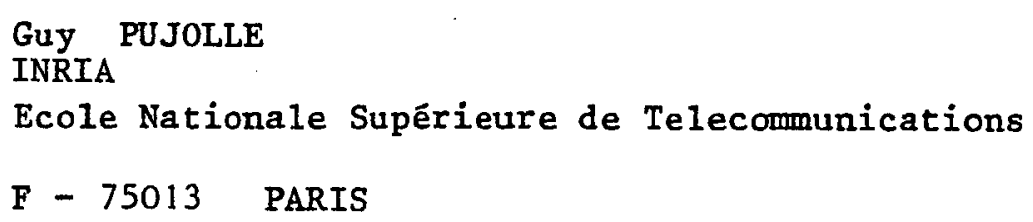




\section{Résumé}

HDIC est un protocole de contrôle des lignes terrestres reconnu par les instances internationales de normalisation. Si on veut utiliser HDLC pour des canaux satellite, on rencontre deux inconvénients majeurs causës par le délai de propagation très élevé: 1 - un blocage du à la limitation de la taille de la fenêtre; 2 - une chute du débit provenant de la politique de reprise sur erreur.

Dans cet article nous présentons un modèle de traitement des erreurs sur des canaux satellite en insistant sur le phẻnomène d'erreurs groupées. Le modèle est utilisé pour expliquer le comportement peu satisfaisant de HDLC sur des canaux satellite.

On propose quelques méthodes pour remedier aux inconvénients pré-cités, notamment en introduisant la notion de 'sous-canal virtuel'. Il s'agit d'un protocole à deux niveaux: le niveau bas (niveau sous-canal) fonctionne suivant le mode HDLC classique; le niveau supérieur est le melange de plusieurs sous-canaux indépendants, d'un mecanisme de réarrangement des trames et d'une politique de contrôle du flux pour le système global.

\section{Abstract}

HDLC is an internationally accepted link control protocol which has been designed for terrestrial links. When applied to satellite links, however, HDLC has two major deficiencies: 1. Blocking due to window size limitations; 2. Throughput degradation due to the fact that at most one reject may be outstanding at a given time. Both problems arise as a consequence of the very long signal propagation delay of satellite links.

In this paper we describe a model for the error behaviour of satellite links with special emphasis on bursty errors. This model is used to explain the unsatisfactorily behaviour of HDLC for satellite links.

Following that, several methods of overcoming these problems are discussed. A new concept ('Virtual Subchannels') is presented which eliminates the HDLC-deficiencies by means of a two-level hierarchy of link protocols. The lower level ('Subchannel level') operates exact Iy according to HDLC; the second level ('Total System') is a superposition of several independent subchannels together with a frame reordering scheme and a flow control rule for the total system. 
1. EDLC : Principles and Problems for Satellite Commuications

During the past few years, HDLC (see $[6],[7]$ ) has become a widely accepted international standard for communication protocols;

it forms the lowest nonphysical level of the well-known Iso-hierarchy (see [9]). The main purpose of this protocol level is to provide a relatively error free transmission of bit streams over insecure channels ( $v i a$ a cyclic redundancy checksum of frame contents and by retransmissions of frames in case of an incorrect checksum) as well as to provide high throughput together with a flow control scheme (both aims are realized by using window sizes W: a sender may increase the throughput by transmitting in advance but he will be blocked after $W$ successive transmissions if he did not receive the acknowledgment for the first one).

If a HDLC-frame is not correctly received due to a loss of information or due to a transmission error which results in a wrong checksum (this will be detected by the receiver at the moment when he correctly receives any later packet since overtaking of frames is impossible on a single link) the receiver asks for retransmissions by using special HDLC-frames:

$-\operatorname{REJ}\left(a_{j}\right) \quad$ : reject of frame $a_{j}$; asks for retransmissions of all the frames beginning with $a_{j}$, i.e. including all the frames which have eventually been sent in advance.

- $\operatorname{SREJ}\left(a_{j}\right)$ : selective reject of frame $a_{j}$; only $a_{j}$ has to be retransmitted. Frames which have been sent in advance and which arrive correctly at the receiver during this time are buffered at the receiver but are not acknowledged.

In the actual version of the recommendation X.25 which contains HDLC as a subset, the possibility SREJ has been cancelled (see [6]).

The performance of REJ and SREJ retransmission strategies have been studied in a few papers $([1][3],[5])$. It turns out that REJ has some disadvantages when the window size $W$ is growing since in this case a large number of packets might have unneccessarily to be retransmitted. On trie other hand, SREJ is not very suitable if there is a substantial probability that a frame will be in error; furthermore SREJ needs more organizational effort than the simpler procedure REJ (buffering at the receiver, ...) and this could have been the main reason for cancelling it in the actual $\times .25$ recomendation. 
In todays applications the links which are controlled by HDLC are very short (terrestrial links); thus message propagation delays are very short, too. The choice of HDLC parameters (maximum window sizes, ...) was certainly dictated by properties of terrestrial links only.

However, things are considerably changing if satellite links are used (i.e. transmission via geostationary satelites, $\approx 36.000 \mathrm{~km}$ above the earth) and if we want to control those links by HDLC-like principles. Satellite links differ from terrestrial ones mainly by the big amount of propagation delay ( $\approx 0.27 \mathrm{sec}$ for $2.36000 \mathrm{~km}$ ); if we include the time necessary for the ACK, the total propagation delay for a frame communication turns out to be 0.54 seconds.

Let $B$ be the bandwidth of satellite links (bis/sec)

" L " " (maximum) length of a frame (bits).

Then

$P:=0.27 \cdot \frac{B}{L}$ is the total number of frames which can be sent until the moment when the first one arrives at the receiver and $\mathrm{P}_{\mathrm{ACK}}:=0.54 \cdot \frac{\mathrm{B}}{\mathrm{L}}$ is the number of frames which may be sent in advance during one 'transmission + acknowledgment'-procedure.

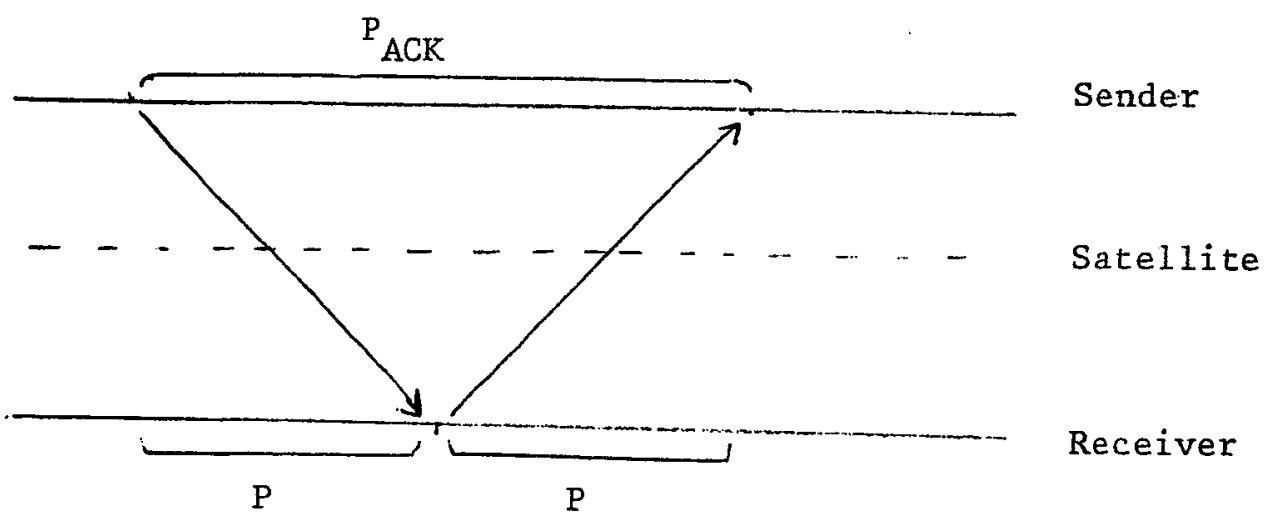

The long propagation delay has severe consequences:

(A) Blocking of senders due to window size limits

In the actual BDLC recommendation, the window size $W$ is limited to $W \leq 7$ (or optionally to $W \leq 127$ ). If the bandwidth, $B$, is high and/or the frames are not excessively long, however, ${ }_{A C K}$ will be much greater than $W$, thus the sender will become unnecessarily blocked even if the link is error free.

(B) Throughput degradation due to transmission errors Due to the large amount of propagation delay, the rejection and the retransmission of an erroneous frame is very time consuming. 
Moreover, since the number of REJ or SREJ frame which are outstanding at any given time is limited by at most $1\left(\operatorname{REJ}\left(a_{j}\right)\right.$ or $\operatorname{SREJ}\left(a_{j}\right)$ automatically acknowledges all the frames preceding $a_{j}$, thus no two such control frames may exist simultaneously) the performance of the usual HDLC procedure will be rather poor for satellite links since the correction of subsequent errors is delayed for a very long time. This problem becomes even more serious in 'bursty systems' when the distance of succesive errors is rather small in many cases.

In section 2 of this paper, we describe a model for simulating errors in bursty systems. This analysis will show that for many satellite systems the restriction of having at most one rejection at any given time has dramatic disadvantages for the throughput. To summarize the considerations of this introductory section we want to point out that the actual HDLC recommendation has the following two major deficiencies when applied to satellite links:

1. the maximum window size, $\mathrm{W}$, is too sma11;

2. the restriction to have at most one rejection at any given time leads to throughput degradation for error prone transmissions.

It has to be said once more that both problems do not appear for terrestrial links, i.e. for the type of links for which HDLC has been originally defined.

In section 3 of the paper we present a new concept (virtual subchannels) which is based on the HDLC procedure but which removes the deficiencies which have been desribed above; it turns out that this protocol results in a much better performance for satellite links than the classical HDLC. The price to be payed is an increased complexity of the protocol (e.g. ability of managing several rejections at a time) but as for example kleinrock pointed out $([2])$ one gets nothing for nothing or 'you have to borrow Peter to pay Paul'. 


\section{A Model for the Error Behaviour of Satellite Links with special Emphasis on Bursty Errors}

Let $A_{0}, A_{1}, \ldots$ be a sequence of bits or of larger entities (i.e. packets) which have to be transmitted over a satellite link in this order.

Let $s_{i} \stackrel{\text { def }}{=} s_{i}^{1}$ denote the event that $A_{i}$ is not correctly received " $\overline{s_{i}}$ def $s_{i}^{0}$ denote the event that $A_{i}$ will be correctly received.

A parameter of interest is the mean distance between consecutive errors, i.e. $\quad E:=\sum_{m=1}^{\infty} \operatorname{mr}\left(s_{m}, \overline{s_{m-1}}, \overline{s_{m-2}}, \ldots, \overline{s_{1}} \mid s_{0}\right)$

Experimental data show (see [8]) that errors do not occur independently of each other but mostly occur in bursts; during certain time periods (due to meteorological or other reasons) the probability of transmission perturbations is very high as compared to the rather low perturbation probability during 'regular periods'.

Thus

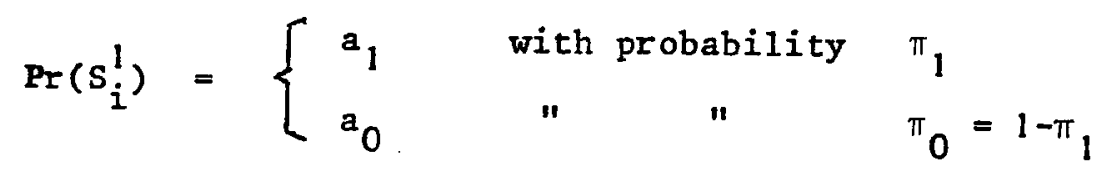

where $a_{1}$ and $a_{0}$ denote the error probabilities in the 'good state' and in the 'bad state' respectively; we assume that the system alternates between good states and bad states such that the proportions of being in these states is given by $\pi_{1}$ and $\pi_{0}$ respectively.

Typical data for the system parameters introduced thus far are the (see[4][8]): $\left.\begin{array}{rlrl}\pi_{1} & \approx 0.99 & \pi_{0} \approx 0.01 & \\ a_{1} \approx 10^{-7} & a_{0} \approx 10^{-4} & \text { (bit level) } \\ a_{1} \approx 10^{-4} & a_{0} \approx 10^{-1} & \text { (packet leve1, packet length } \\ & & & 1000 \text { bits) }\end{array}\right)$

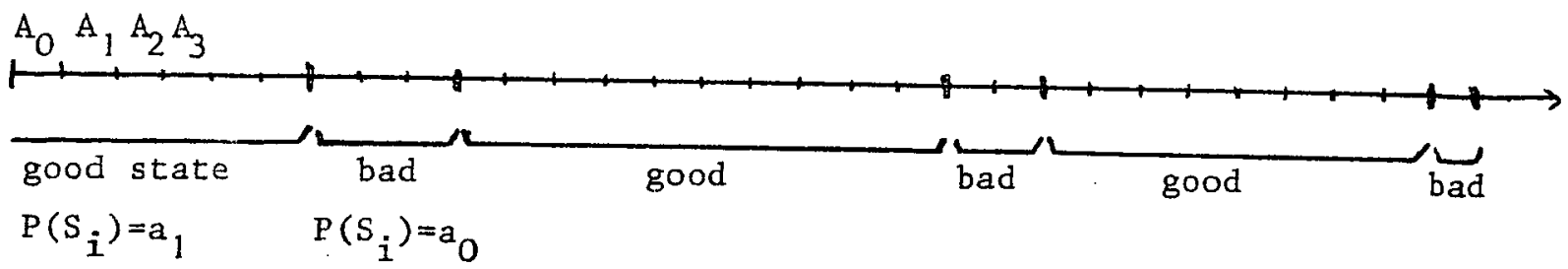


A very important parameter is the 'burstiness' of the system; this parameter characterizes the average duration of good states and bad states respectively. In the following we develop a model to represent several kinds of bursty systems:

To characterize the actual state of the system, we introduce a sequence $x_{0}, x_{1}, \ldots$ where $x_{i}$ is associated to the transmission of $A_{i}$. $x_{i}$ is a random variable which takes only two possible values 1 or 0 depending on whether the system is in the good state or in the bad state. Let $x_{i}^{1}$ denote the event that $x_{i}=1, i . e$. that we are in the 'good state', " $x_{i}^{0} "$ " " $x_{i}=0$, i.e. " " "bad state'.

We are now ready to introduce our main model assumption:

The sequence $\mathrm{x}_{0}, \mathrm{X}_{1}, \ldots$ of rand om variables forms a homogeneous two-state Markov-chain; thus:

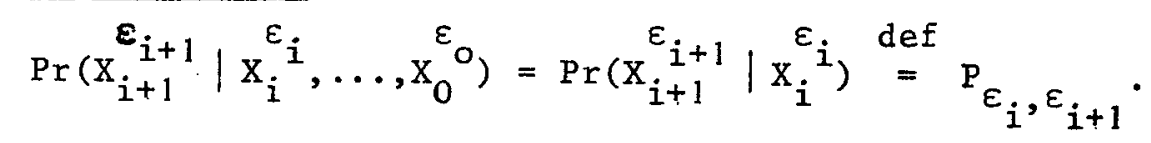

The transition matrix of this Markov-chain is given by:

$$
\pi=\left(\begin{array}{ll}
p_{0,0} & p_{0,1} \\
p_{1,0} & p_{1,1}
\end{array}\right) \quad \operatorname{def}\left(\begin{array}{cc}
1-p & p \\
s & 1-s
\end{array}\right)
$$

The stationary solution for the state probabilities exists and is given by:

$$
\begin{aligned}
& \pi_{0}=\lim _{i \rightarrow \infty} \operatorname{Pr}\left(x_{i}^{0}\right)=\frac{s}{p+s} \\
& \pi_{1}=\lim _{i \rightarrow \infty} \operatorname{Pr}\left(x_{i}^{1}\right)=\frac{p}{p+s}
\end{aligned}
$$

Thus we see that the transition probabilities $p$ and $s$ (rates of changes from bad state to good state and vice versa) cannot be chosen independently but are related to each other by means of:

$$
s=\frac{\pi_{0}}{\pi_{1}} \cdot p \text {. }
$$

The parameter $p$ characterizes the burstiness of the system:

$$
\begin{aligned}
& \mathrm{p} \rightarrow 0: \text { 'Very bursty systems'; the average duration of a good state } \\
& \text { as well as of a bad state is very high; } \\
& \mathrm{p} \rightarrow 1: \text { 'Non-bursty behaviour'; the average duration of bad states } \\
& \text { tends towards } 1 \text {. This system may be characterized by } \\
& \text { independent errors which occur with a mixed probability } \\
& \quad \operatorname{Pr}\left(\mathrm{s}_{i}\right)=\mathrm{a}^{*}=\mathrm{a}_{0} \cdot \pi_{0}+\mathrm{a}_{1} \cdot \pi_{1} .
\end{aligned}
$$


Thus our model is described by the following system parameters:

1. $a_{1}=\operatorname{Pr}\left(\mathbf{s}_{i} \mid \mathbf{x}_{i}^{1}\right)$

2. $a_{0}=\operatorname{Pr}\left(s_{i} \mid x_{1}^{0}\right)$

3. $\mathrm{p}=\operatorname{Pr}\left(\mathrm{x}_{i+1}^{1} \mid \mathrm{x}_{i}^{0}\right)$

4. $\pi_{0}=\lim _{i \rightarrow \infty} \operatorname{Pr}\left(x_{i}^{0}\right)$ error probability in the good state

$"$

$"$

" " bad state

burstiness parameter; rate of changing

from bad state to good state.

steady state probability of being in the bad state.

All the other parameters introduced so far are now obtained by simple relations:

$\pi_{1}=1-\pi_{0} \quad$ steady state probability of being in the good state.

s. $=\frac{\pi_{0}}{\pi_{1}} \cdot p$ rate of changing from good state to bad state.

$c=\frac{\pi_{0}}{\pi_{1}} \quad$ relation between 'bad state' and 'good state' probabilities.

In the following, we will use $c$ instead of $\pi_{0}$ as an independent system parameter; $\pi_{0}$ then is obtained by $\pi_{0}=\frac{c}{1+c}$.

Calculation of the average distance between successive errors:

In this section we develop an expression for the average distance

$$
E:=\sum_{m=1}^{\infty} m \cdot \operatorname{Pr}\left(s_{m}, \overline{s_{m-1}}, \ldots, \overline{s_{1}} \mid s_{0}\right)
$$

for very bursty systems (i.e. for $\mathrm{p} \rightarrow 0$ ). For this reason the formula will be exact up to a term of order $O\left(p^{2}\right)$.

If the behaviour of the system is nonbursty (independent errors occuring with probability $a^{*}$ ) the average distance is simply given by:

$$
E=\sum_{m=1}^{\infty} m \cdot \operatorname{Pr}\left(s_{m}, \overline{s_{m-1}}, \ldots, \overline{s_{1}}\right)=\sum_{m=1}^{\infty} m \cdot a^{*} \cdot\left(1-a^{*}\right)^{m-1}=\frac{1}{a^{\star}} .
$$

First of all we have to derive an expression for

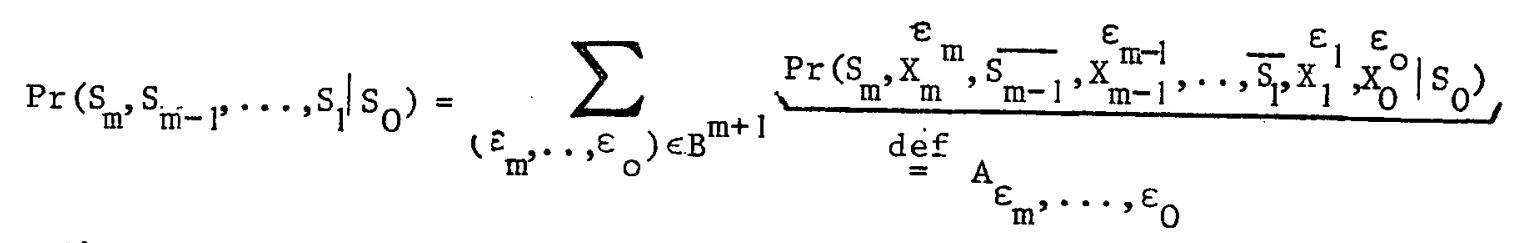

According to our model assumptions (the sequence $\left(x_{i}\right)$ forms a Markov chain) the probability $A_{\varepsilon_{m}}, \ldots, \varepsilon_{0}$ is given by: 


$$
\begin{aligned}
& A_{\varepsilon_{m}}, \ldots, \varepsilon_{0}=\operatorname{Pr}\left(\mathrm{s}_{m} \mid \mathrm{x}_{\mathrm{m}}^{\varepsilon_{\mathrm{m}}}, \ldots\right) \cdot \operatorname{Pr}\left(\mathrm{x}_{\mathrm{m}}^{\varepsilon_{\mathrm{m}}} \mid \overline{\mathrm{s}}_{\mathrm{m}-1}, \mathrm{x}_{\mathrm{m}-1}^{\varepsilon_{\mathrm{m}-1}}, \ldots\right) \\
& \cdot \operatorname{Pr}\left(\overline{\mathrm{S}}_{\mathrm{m}-1} \mid \mathrm{x}_{\mathrm{m}-1}^{\varepsilon_{\mathrm{m}-1}}, \ldots\right) \cdot \operatorname{Pr}\left(\mathrm{x}_{\mathrm{m}-1}^{\varepsilon_{\mathrm{m}-1}} \mid \overline{\mathrm{s}}_{\mathrm{m}-2}, \mathrm{x}_{\mathrm{m}-2}^{\varepsilon_{\mathrm{m}-2}}, \ldots\right) \\
& \text { - } \cdots \cdot \operatorname{Pr}\left(\overline{\mathrm{s}_{1}} \mid \mathrm{x}_{1}^{\varepsilon_{1}}, \mathrm{x}_{\mathrm{o}}^{\varepsilon_{0}}, \mathrm{~s}_{\mathrm{o}}\right) \cdot \operatorname{Pr}\left(\mathrm{x}_{1}^{\varepsilon_{1}} \mid \mathrm{x}_{\mathrm{o}}^{\varepsilon_{0}}, \mathrm{~s}_{\mathrm{o}}\right) \cdot \operatorname{Pr}\left(\mathrm{x}_{\mathrm{o}}^{\varepsilon_{0}} \mid \mathrm{s}_{0}\right)
\end{aligned}
$$

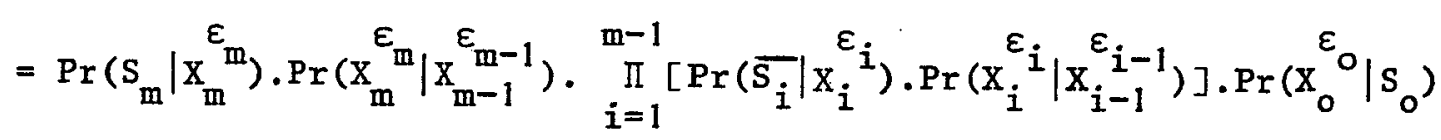

$$
\begin{aligned}
& =a_{\varepsilon_{m}} \cdot p_{\varepsilon_{m-1}}, \varepsilon_{m} \cdot \prod_{i=1}^{m-1}\left(1-a_{\varepsilon_{i}}\right) \cdot p_{\varepsilon_{i-1}}, \varepsilon_{i} \cdot \frac{a_{\varepsilon_{0}} \cdot \pi_{\varepsilon_{0}}}{a_{0} \pi_{0}+a_{1} \pi_{1}} \\
& =a_{\varepsilon_{m}} \cdot p_{\varepsilon_{m-1}}, \varepsilon_{m} \cdot \prod_{i=1}^{m-1}\left(1-a_{\varepsilon_{i}}\right) \cdot p_{\varepsilon_{i-1}}, \varepsilon_{i} \cdot \frac{{ }^{a} \varepsilon_{0} \cdot\left(c+\operatorname{sign}\left(\varepsilon_{0}\right) \cdot(1-c)\right.}{a_{0} \cdot c+a_{1}}
\end{aligned}
$$

since

$$
\pi_{0}=\frac{c \cdot p}{p+s}, \quad \pi_{1}=\frac{p}{p+s}
$$

hence : $\pi_{\varepsilon_{0}}=\left(c+\operatorname{sign}\left(\varepsilon_{0}\right) \cdot(1-c)\right) \cdot \frac{p}{p+s}$.

Thus we obtain the following formula for $\operatorname{Pr}\left(\mathrm{s}_{\mathrm{m}}, \overline{\mathrm{s}_{\mathrm{m}-1}}, \ldots, \overline{\mathrm{s}_{1}} \mid \mathrm{s}_{0}\right)$ :

$$
\begin{aligned}
\operatorname{Pr}\left(S_{m}, \overline{S_{m-1}}\right. & \left.\ldots, \widehat{S_{1}} \mid S_{0}\right) \\
& =\frac{1}{a_{0} \cdot c+a_{1}} \cdot\left(T_{(0,0)}+T_{(0,1)}+T_{(1,0)}+T_{(1,1)}\right)
\end{aligned}
$$

where $T_{(\alpha, \beta)}$ is given by :

$$
T_{(\alpha, \beta)}=\left(a_{0} \cdot c+a_{1}\right) \cdot \sum_{\left\{\left(\varepsilon_{m}, \ldots, \varepsilon_{0}\right) \in B^{m-1} \mid\left(\varepsilon_{m}, \varepsilon_{m-1}\right)=(\alpha, \beta)\right\}}^{A} A_{m}, \ldots, \varepsilon_{0}
$$

The number of terms forming $\mathrm{T}_{(}(\alpha, \beta)$ increases exponentially with $\mathrm{m}$; we are, however, only interested in a closed formula which is valid up to terms of order $\mathrm{p}^{2}$ or higher ; thus all the summands which contain at least twice the factor $p$ may be neglected; this results in : 


$$
\begin{aligned}
& \mathrm{T}_{(0,1)}=\mathrm{a}_{0} \cdot \mathrm{p}_{1,0} \cdot \prod_{i=1}^{\mathrm{m}-1}\left(1-\mathrm{a}_{1}\right) \cdot \mathrm{p}_{1,1} \cdot \mathrm{a}_{1}+\boldsymbol{\theta}_{\left(\mathrm{p}^{2}\right)}=\mathrm{a}_{0} \mathrm{a}_{1} \mathrm{cp} \cdot\left[\left(1-\mathrm{a}_{1}\right)(1-\mathrm{cp})\right]^{\mathrm{m}-1}+\Theta_{\left(p^{2}\right)}
\end{aligned}
$$

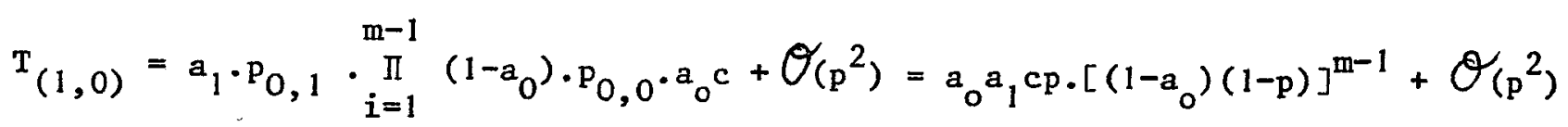

$$
\begin{aligned}
& { }^{T}(0,0)=a_{0} \cdot(1-p) \cdot\left\{a_{0} c \cdot\left[\left(1-a_{0}\right)(1-p)\right]^{m-1}+c p\left(1-a_{0}\right) \cdot a_{1} \cdot\left[\left(1-a_{0}\right)(1-p)\right]^{m-2} \cdot \frac{1-\beta^{m-1}}{1-\beta}\right\} \\
& +\theta\left(p^{2}\right) \\
& T_{(1,1)}=a_{1} \cdot(1-c p) \cdot\left\{a_{1} \cdot\left[\left(1-a_{1}\right) \cdot(1-c p)\right]^{m-1}+c p a_{0}\left(1-a_{1}\right) \cdot\left[\left(1-a_{1}\right)(1-c p)\right]^{m-2}\right. \text {. } \\
& \frac{1-\left(\beta^{-1}\right)^{m-1}}{1-\beta^{-1}}+\theta\left(p^{2}\right) \\
& \text { where : } \quad \beta=\frac{\left(1-a_{1}\right)(1-c p)}{\left(1-a_{0}\right)(1-p)}
\end{aligned}
$$

The formulae obtained for $\mathrm{T}_{(0,1)}$ and for $\mathrm{T}(1,0)$ are obvious; the derivation of the expressions for $\mathrm{T}(0,0)$ and for $\mathrm{T}_{(1,1)}$ is given in the appendix.

The expected value between successive errors is now given by

$$
\begin{aligned}
E & =\sum_{m=1}^{\infty} m \cdot \operatorname{Pr}\left(S_{m}, \overline{S_{m-1}}, \overline{s_{m-2}}, \ldots, \overline{s_{1}} \mid s_{0}\right) \\
& \approx \frac{1}{1+\frac{a_{1}}{a_{0} \cdot c}} \cdot\left\{\frac{a_{0} \cdot(1-p)+\frac{2 p a_{1}}{1-\beta}}{\left[1-\left(1-a_{0}\right)(1-p)\right]^{2}}+\frac{\frac{a_{1}^{2}}{a_{0} c} \cdot(1-c p)+\frac{2 p a_{1}}{1-\frac{1}{\beta}}}{\left[1-\left(1-a_{1}\right)(1-c p)\right]^{2}}\right\}
\end{aligned}
$$

For very bursty systems $(p \rightarrow 0)$ the following expressions are obtained :

$$
\begin{aligned}
E & \underset{(p \rightarrow 0)}{\longrightarrow} \frac{1}{1+\frac{a_{1}}{a_{0} \cdot c}} \cdot\left(\frac{a_{0}}{\left(1-\left(1-a_{0}\right)\right)^{2}}+\frac{\frac{a_{1}^{2}}{a_{0} c}}{\left(1-\left(1-a_{1}\right)\right)^{2}}\right) \\
& =\frac{1+c}{a_{0} c+a_{1}}= \begin{cases}\frac{1}{a_{1}} & \text { if } c=0 \quad \text { (i.e. good state only) } \\
\frac{1}{a_{0}} & \text { if } a_{0}=a_{1} \\
\approx \frac{1}{a_{0} c}<\frac{1}{a_{1}} & \text { if } a_{1} / a_{0} \ll c<1\end{cases}
\end{aligned}
$$

Thus we see that in the very important case " $a_{1} / a_{0} \ll c$ " the mean distance between errors is dictated by the 'bad state probability' $a_{0}$ rather than by the 'good state error probability' $a_{1}$. 
Expected distance between consecutive errors given that the first error occured in the bad state.

It will be shown that this expectation is entirely dictated by the bad state probability $a_{0}$ in buraty systems.

We have to calculate

$$
E^{*} \stackrel{\text { def }}{=} \sum_{m=1}^{\infty} m \cdot \operatorname{Pr}\left(s_{m}, \overline{s_{m-1}}, \overline{s_{m-2}}, \ldots, \overline{s_{1}} \mid s_{0}, x_{0}^{0}\right)
$$

The terms of this sum are given by :

$$
\begin{aligned}
& \operatorname{Pr}\left(\mathrm{s}_{\mathrm{m}}, \overline{\mathrm{s}_{\mathrm{m}-1}}, \ldots, \overline{\mathrm{s}_{1}} \mid ' \mathrm{~s}_{0}, \mathrm{x}_{0}^{0}\right) \\
& =\sum_{\left(\varepsilon_{\mathrm{m}}, \ldots, \varepsilon_{1}\right) \in \mathrm{B}^{\mathrm{iI}}} \operatorname{Pr}\left(\mathrm{s}_{\mathrm{m}}, \mathrm{x}_{\mathrm{m}}^{\varepsilon_{\mathrm{m}}}, \overline{\mathrm{s}_{\mathrm{m}-1}}, \ldots, \mathrm{s}_{1}, \mathrm{x}_{1}^{\varepsilon_{1}} \mid \mathrm{x}_{0}^{0}, \mathrm{~s}_{0}\right)
\end{aligned}
$$

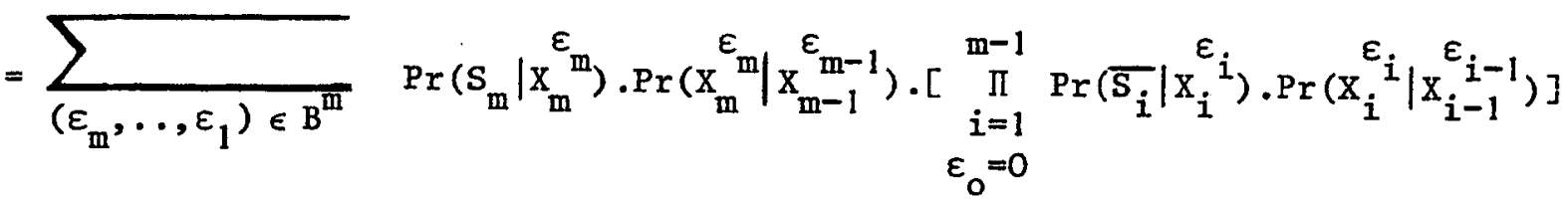

$$
\begin{aligned}
& \text { m-1 }
\end{aligned}
$$

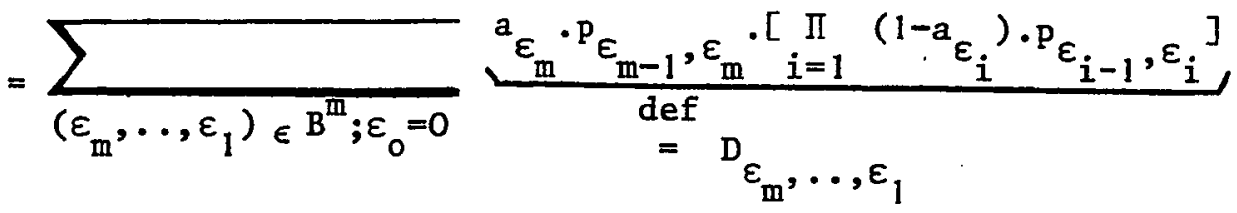

$$
\begin{aligned}
& =T_{(0,0)}^{\prime}+T_{(0,1)}^{\prime}+T_{(1,0)}^{\prime}+T_{(1,1)}^{\prime} \\
& \text { where } \quad T_{(\alpha, \beta)}^{\prime}:=\sum_{\left(\varepsilon_{\mathrm{m}}, \ldots, \varepsilon_{1}\right) \in \mathrm{B}^{\mathrm{II}} ; \varepsilon_{\mathrm{o}}=0} \mathrm{D}_{\varepsilon_{\mathrm{m}}, \ldots, \varepsilon_{1}} \\
& \left(\varepsilon_{\mathrm{m}}, \varepsilon_{\mathrm{m}-1}\right)=(\alpha, \beta)
\end{aligned}
$$

Once again, we are only interested in formulae which are valid up to terms of order $p^{2}$. Due to the restriction $\varepsilon_{0}=0$, the expressions for $\mathrm{T}^{\prime}(\alpha, \beta)$ are considerably simplified as compared with the expressions for $T_{(\alpha, \beta)}$. We obtain :

$$
\begin{aligned}
& T_{(0,0)}^{\prime}=a_{0} \cdot p_{0,0} \cdot \prod_{i=1}^{m-1}\left[\left(1-a_{0}\right) p_{00}\right]+\theta\left(p^{2}\right)=a_{0}(1-p) \cdot\left[\left(1-a_{0}\right)(1-p)\right]^{m-1}+\theta\left(p^{2}\right) \\
& T_{(0,1)}^{\prime}=\theta\left(p^{2}\right) \\
& T_{(1,0)}^{\prime}=a_{1} \cdot p_{0,1} \cdot \prod_{i=1}^{m-1}\left[\left(1-a_{0}\right) p_{00}\right]+\theta\left(p^{2}\right)=a_{1} p \cdot\left[\left(1-a_{0}\right)(1-p)\right]^{m-1}+\theta\left(p^{2}\right)
\end{aligned}
$$




$$
\begin{array}{r}
T_{(1,1)}^{\prime}=a_{1} p \cdot\left[\left(1-a_{1}\right) \cdot(1-c p)\right]^{m-1} \cdot \frac{1-\left(\frac{1}{\beta}\right)^{m-1}}{1-\frac{1}{\beta}} \\
\text { where } \beta=\frac{\left(1-a_{1}\right)(1-c p)}{\left(1-a_{0}\right)(1-p)} .
\end{array}
$$

The derivation of the formula for $T_{(1,1)}$ is given in the appendix.

By inserting these expressions we obtain the following result for the probability that the distance between consecutive errors is exactly $m$ :

$$
\begin{aligned}
& \operatorname{Pr}\left(s_{m}, \widetilde{s_{m-1}}, \ldots, \widetilde{s_{1}} \mid s_{0}, x_{0}^{0}\right) \\
& \quad=\left(a_{0}(1-p)+\frac{a_{1} \cdot p}{1-\beta}\right) \cdot\left[\left(1-a_{0}\right)(1-p)\right]^{m-1}+\frac{a_{1} \cdot p}{1-\frac{1}{\beta}} \cdot\left[\left(1-a_{1}\right)(1-c p)\right]^{m-1}+\theta_{\left(p^{2}\right.}{ }^{2}
\end{aligned}
$$

Remark: This formula is exact in the following cases:

$$
\begin{aligned}
& -p=0: \operatorname{Pr}\left(s_{m}, \overline{s_{m-1}}, \ldots, \overline{s_{1}} \mid s_{0}, x_{0}^{0}\right)=a_{0} \cdot\left(1-a_{0}\right)^{m-1} \\
& -m=1: \operatorname{Pr}\left(s_{1} \mid s_{0}, x_{0}^{0}\right)=a_{0} \cdot(1-p)+a_{1} \cdot p .
\end{aligned}
$$

Finally, the average distance between errors given that the first error occurer in the bad state is now obtained as:

$$
\begin{aligned}
& \mathrm{E}^{*}=\sum_{\mathrm{m}=1}^{\infty} \mathrm{m} \cdot \operatorname{Pr}\left(\mathrm{s}_{\mathrm{m}}, \overline{\mathrm{s}_{\mathrm{m}-1}}, \ldots, \overline{\mathrm{s}_{1}} \mid \mathrm{s}_{0}, \mathrm{x}_{0}^{0}\right) \\
& \approx \frac{a_{0} \cdot(1-\mathrm{p})+\frac{\mathrm{a}_{1} \mathrm{p}}{1-\beta}}{\left[1-\left(1-\mathrm{a}_{0}\right)(1-\mathrm{p})\right]^{2}}+\frac{\frac{a_{1} p}{1-1 / \beta}}{\left[1-\left(1-a_{1}\right)(1-c p)\right]^{2}} \\
& \underset{(p \rightarrow 0)}{\longrightarrow} \frac{1}{a_{0}} .
\end{aligned}
$$

This last result is very important; it proves that in bursty systems the mean distance between consecutive errors is rather small. In the protocol HDLC : however, at most one REJ or SREJ may be outstanding at a time. Thus due to the extremely long propagation delay it takes a very long time to correct error bursts; during this time the transmission of new frames will be blocked due to the window size limitations and due to retransmissions. These effects lead to severe throughput degradations which cannot be tolerated in general. 


\section{The Virtual Subchannel Concept for Satellite Transmissions}

The major deficiencies of HDLC for satellite communications are (see 1.):

1. Window size $W \leq 127$, thus mostly $W \ll P_{A C K}$, hence senders become blocked even if the channel is error free.

2. At most one rejection may be outstanding at any given time; thus in case of transmission errors the throughput will be very seriously affected.

Several possibilities can be discussed to solve these problems;

in the following, we discuss four of them:

A. Increase of the window size $W$ to $W^{\prime}$ such that $W^{\prime}>P_{A C K}$. The inconvenient of this remedy is that a larger number of frames (up to $W^{\prime}$ frames) have to be retransmitted in case of a REJ-frame.

$B$. Increase of the maximum frame length $L$, thus decreasing $P_{A C K}=0.54 \cdot \frac{B}{L}$. This solution is particularly simple, but the probability that a frame is in error will be increasing as well (approximately linearly with the frame length $\mathrm{L}$ ); furthermore, internal fragmentation of frames could become an important factor.

C. Several rejections at a time, change from SREJ to REJ: This interesting possibility has been investigated for the experimental IBM satellite link: This protocol version uses SREJ first, but if during such a correction procedure another error is detected by the receiver, a REJ-frame will be sent which replaces SREJ. The motivation of this procedure seems to be quite intuitive: as long as errors are rare, SREJ is better than REJ (see [5]), but if errors become more likely, the performance of REJ will become better; in the IBM procedure the existence of a second error during SREJ is used as an indicator for an increased error probability, thus supporting a change from SREJ to REJ. Obviously, at any given time two simultaneous rejections may coexist. This solution seems to be very efficient; it is easy to implement. Unf ortunately, it is not in accordance with the existing and internationally adopted standard HDLC. 
D. Virtual Subchannels

In this concept we imagine that transmissions are effected by $M$ virtual subchannels $C_{0}, \ldots, C_{M-1}$ which are used in cyclic order; thus $C_{i \bmod M}$ is responsible for the transmission of frame $a_{i}$.

In reality, all the frames $a_{0}, a_{1}, \ldots, a_{i}, \ldots$ are transmitted over the same satellite link; the subchannel concept, however, gives us some freedom in designing larger window sizes and better retransmission strategies; this will be shown in the following. Each subchannel has a HDLC window size $W$ (i.e. $W \leqslant 127$ ). Thus up to $W$ frames may be unacknowledged per subchannel; W buffers are necessary per subchannel to hold these frames ( both ath the senders and at the receivers end ). If a frame is rejected by subchannel $c_{k}$, only frames $a_{j}$ where $j=k \bmod M$ are possibly retransmitted.

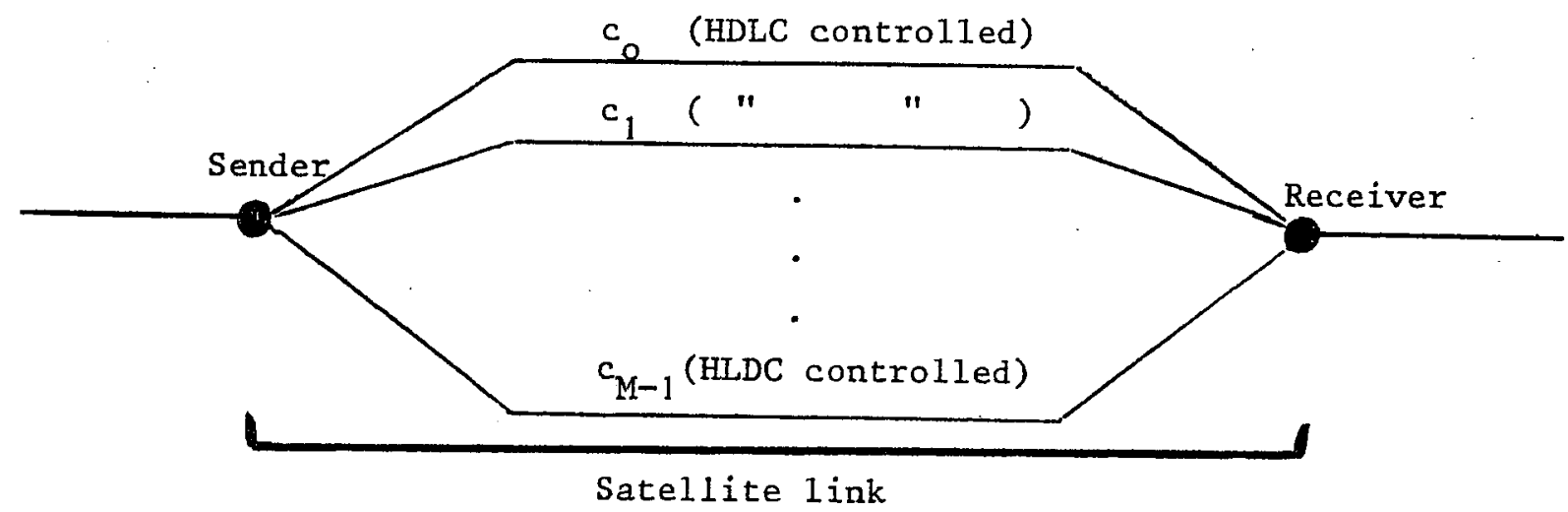

Consequences of the virtual subchannel concept:

1. Each subchannel operates exactly according to the HDLC-recommendation and may be controlled either by REJ or by SREJ principles; different subchannels might even apply different strategies (possibly REJ for subchannels $C_{0}, \ldots, C_{r}$, SREJ for $C_{r+1}, \ldots, C_{M-1}$ ). We might even use different window sizes $W_{i}$ for the subchannels $C_{i}$.

2. The subchannel concept 'simulates' a total window size $W^{*}$ :

$$
W^{*}=\sum_{i=0}^{M-1} W_{i} \quad\left(W_{i=W}=W \cdot M,\right.
$$

but the number of frames to be retransmitted due to a REJ by subchannel $C_{i}$ does never exceed $W_{i}$.

Thus the total system implicitly uses a SREJ-concept (even if REJ is used for each subchannel) since only 'selected' frames have to be retransmitted in case of an error. For this reason, $W^{*}=W \cdot M$ buffers are necessary also at the receivers end (as with the conventional SREJ method using a window size $W^{*}$ ). 
3. Since subchannels are controlled independently of each other on a tDLC-basis, a frame rejection may exist at any time per subchanne1; thus in the total system, up to $M$ rejections may exist simultaneously. Clearly, the ability of managing several rejections at a time leads to an increase of the organizational complexity of the protocol.

4. The time between subsequent messages which are sent over a given subchannel increases linearly with the total number, $M$, of channels. Thus the detection of a frame error (which is effected by the following correctly received frame of the same subchannel) needs more time than in the classical HDLC concept. This indicates that there should exist an optimal number of subchannels (not too small in order to allow several rejections at a time and to reduce blocking effects, not to large in order to reduce the complexity as well as the detection time of an error).

5. Since subchannel operate independently of each other, a reordering of frames becomes necessary at the receivers end. For the very same reason, we also need a stopping rule at the senders end since otherwise the malfunctioning of one subchannel would necessitate an unlimited number of buffers at the receivers end (the receiver can only free a frame buffer if al1 the predecessors of this frame have been correctly received which will never happen if one of the subchannels is thalfunctioning).

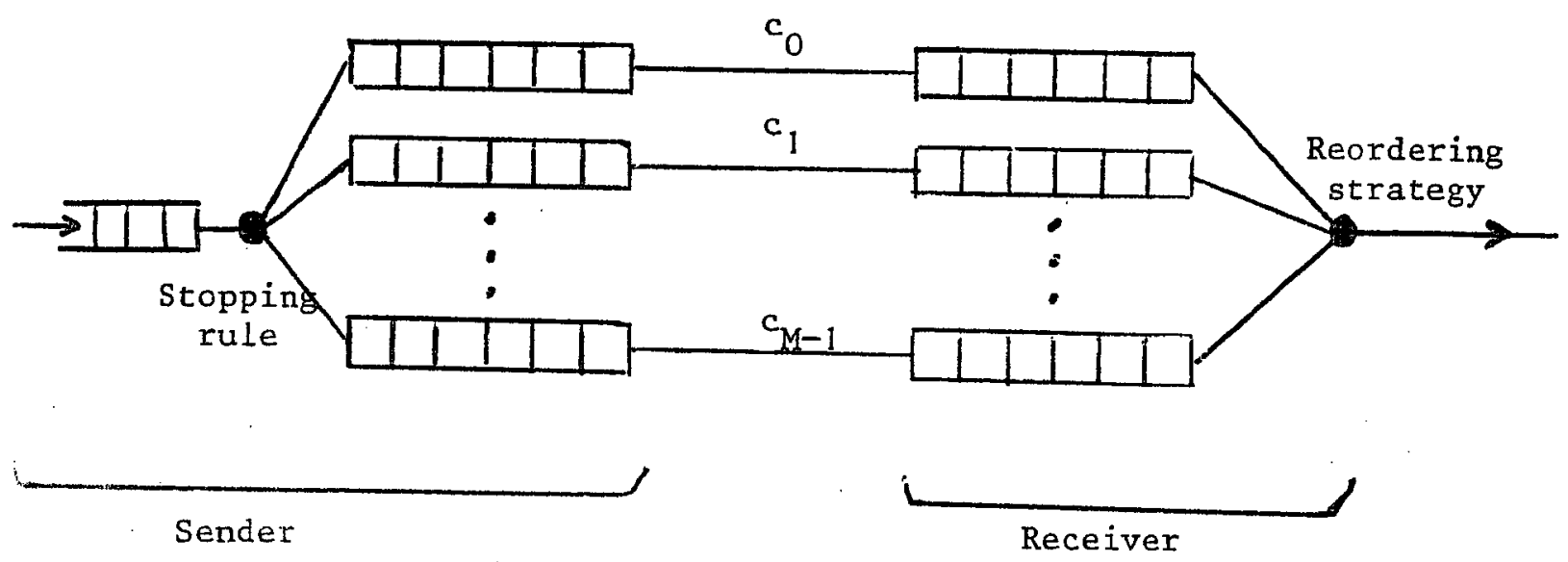

It $\mathrm{x} s \mathrm{~s}$ to be mentioned that such a stopping rule has a very important side effect since it reduces the number of frames which may coexist on a satellite link (including the buffers and the receivers end); thus the stopping rule is also necessary for flow control reasons. 


\section{Definition of the Stopping Rule:}

To define a stopping rule for the total system we have to guarantee that the blocking of one (HDLC controlled) subchannel necessarily leads to the blocking of the total system; thus we postulate that the total system becomes blocked when the next frame is destinated for transmission over an actually blocked subchannel.

This blocking rule defines a two-level protocol for satellite links:

Level 1 (subchanne1 leve1):

This level is exactly in accordance to the classical HDLC.

Level 2 (total system level):

At this level we see a total window size $W^{*}=W \cdot M$. The rejection strategy is a combination of several rejection principles of leve 1 . This level contains also a flow control rule at the cender and a frame reordering scheme at the receiver. As a consequence of this definition of the Stopping Rule, we see that the total system blocks if and only if one of the subchannel blocks; it is impossible that several of the subchannels are blocked at the same time.

System blocking

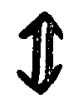

$W^{*}=W^{*} M$ frames sent in advance without ACK

Subchannel blocking

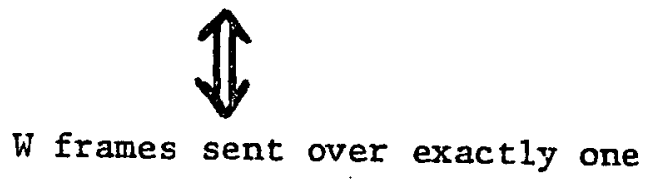
subchannel $C_{k}$ without $A C K$; next frame is also destinated for transmission over $\mathrm{C}_{\mathrm{k}}$.

Thus the total system becomes blocked only after $W^{*} \gg W$ unacknowledged transmissions; this effect leads to a substantial reduction of blocking situations for satellite communications. Fur thermore, several rejections may be outstanding at any given time; thus the throughput of the system will be much better than with the lassical HDLC protocol which only allows one rejection at a time.

As a conclusion we see that the virtual subchannel concept removes both major deficiencies of HDLC for satellite communications by introducing a two-level concept whereby the lower level is exactly in accordance with HDLC. 


\section{Definition of a reordering strategy:}

Reordering will be combined with buffer freeing at the receivers end. The corresponding strategy will be called BES (buffer emptying strategy).

A receiver buffer (containing frame $a_{j}$ ) can be freed if and only if all the frames $a_{0}, \ldots, a_{j}$ have been correctly received. Thus, whereas subchannels operate independent of each other, the BES has to reflect the state of all subchannels.

A possible realization of BES is described in the following:

BES is realized by $M$ concurrent programs for synchronization (one program for each of the subchannels). Programs use additional registers $\mathrm{R}_{0}, \ldots, \mathrm{R}_{M-1}$ where $\mathrm{R}_{i}$ contains the frame number modulo $\mathrm{W}^{*}$ which subchannel $C_{i}$ is waiting for most urgently; $R_{i}$ are private registers for $C_{i}$. Furthermore, a commonly accessible register $T$ will be used which contains the number (modulo $W^{*}$ ) of the frame whose buffer is next to be freed at the receiver; at the beginning we have $R_{i}=i$ and $T=0$.

Control is passed from one subchannel to the next one (i.e. from $C_{i}$ to $C_{i+1} \bmod M$ ) after freeing one subchannel buffer. This will be effected by a vector $\left(b_{0}, \ldots, b_{M-1}\right)$ of binary digits where

$$
b_{i}=\left\{\begin{array}{lll}
1 & \Leftrightarrow & c_{i} \text { has actually the control } \\
0 & \Leftrightarrow & \text { otherwise }
\end{array}\right.
$$

At the beginning, control is with subchannel $C_{0}$, thus $\left(b_{0}, \ldots, b_{M-1}\right)=(1,0, \ldots, 0)$.

The piece of program which has to be executed 'by subchannel $c_{k}$ ' is given in the following:

A. Wait until $b_{k}=1$;

B. If $R_{k}=T$ then goto $C 1$ else goto $C 2$;

C1. (frame is not available).

Wait until the frame arrives (i.e. until $R_{k} \neq T$ ); goto $C 2$;

C2. (frame is available).

Free the buffer containing frame $a_{n}$ where $\left(n \bmod W^{*}\right)=T$;

(this is necessarily the'oldest' frame which has been correctly reseived by $C_{k}$ but whose buffer has not yet been freed).

D. $T:=T+1 \bmod W^{*} ; b_{k+1} \bmod M:=1 ; b_{k}:=0 ;$ goto $A$;

During the execution of these programs, the contents of registers $R_{k}$ may be changed due to the correct reception of frames via $C_{k}$. 


\section{Conclusion and Open Problems}

It has been shown that control procedures (such as HDLC) which are designed for terrestrial links are unacceptable for satellite communications due to blocking effects and throughput degradations. Thus modifications of HDLC are necessary for satellite systems.

The 'Virtual Subchannel' concept has been introduced in this paper: it consists of a two-level hierarchy of protocols:

level 1: $M$ independent virtual subchannels of the communication link assigned for transmission in cyclic order and controlled by the usual HDLC procedure;

level 2: superposition of the subchannels together with a flow control rule at the sender and a frame resequencing rule at the receiver.

Increasing the number, $M$, of subchannels while keeping constant the window size, $W$, per subchannel has the following effects:

\section{Advantages}

A larger total window size becomes available for level two;

Reduction of blocking situations

Throughput increase due to the ability of managing several rejections at the same time

\section{Disadvantages}

More buffers are needed at the sender and at the receiver Detection of frame errors needs more time Organizational complexity of of managing several rejections

Thus one has to be very cautious when determining the number of virtual subchannels. A comparison of advantages and disadvantages indicates that there should exist an 'optimal number' of subchannels (with respect to thoughput and/or waiting times) which is situated in the interval $\left[2: M^{x}\right]$ where $M^{*}$ is a small integer number. 
APPENDIX : Calculation of $T_{(0,0)}, T_{(1,1)}$ and $T_{(1,1)}^{\prime} \quad$ (section 2). Since we neglect terms of order $\mathrm{p}^{2}$ or higher, we have only to consider sequence $\left(\varepsilon_{\mathrm{m}}, \ldots, \varepsilon_{0}\right)$ which contain at most one ' 01 ' or '10' change since such changes occur with probability $p$ or $\mathrm{cp}$ respectively.

Thus :

$$
\begin{aligned}
& \mathrm{T}_{(0,0)}=\mathrm{a}_{\theta} \cdot \underbrace{\mathrm{p}_{0,0}}_{1-\mathrm{p}, 0} \cdot \sum_{\left(\varepsilon_{\mathrm{m}}, \ldots, \varepsilon_{0}\right)=(0,0, \ldots, 1, \ldots, 1)}\left[\prod_{i=1}^{m-1}\left(1-\varepsilon_{\varepsilon_{i}}\right) \cdot p_{\varepsilon_{i-1}, \varepsilon_{i}}\right] \times \\
& (0 \leq \mathrm{k} \leq \mathrm{m}-1) \\
& \begin{array}{c}
{ }_{\varepsilon_{0}} \cdot\left(c+\operatorname{sign}\left(\varepsilon_{0}\right) \cdot(1-c)\right)+\theta_{\left(p^{2}\right)} \\
=a_{0} \cdot(1-p) \cdot\left\{\left[\left(1-a_{0}\right) p_{00}\right]^{m-1} \cdot a_{0} c+\sum_{k=1}^{m-1}\left[\left(1-a_{1}\right) p_{11}\right]^{k-1} \times\right. \\
\left.\left.\left(1-a_{0}\right) p_{10} \cdot\left[\left(1-a_{0}\right) p_{00}\right]^{m-1-k} \cdot a_{1}\right\}+\theta_{(p}{ }^{2}\right)
\end{array} \\
& =a_{0} \cdot(1-p) \cdot\left\{a_{0} c\left[\left(1-a_{0}\right)(1-p)\right]^{m-1}+c p\left(1-a_{0}\right) a_{1} \cdot\left[\left(1-a_{0}\right)(1-p)\right]^{m-2} \times\right. \\
& \left.\frac{1-\beta^{m-1}}{1-\beta}\right\}+\Theta\left(p^{2}\right) \\
& \text { where } B=\frac{\left(1-a_{1}\right)(1-c p)}{\left(1-a_{0}\right)(1-p)} \text {. }
\end{aligned}
$$

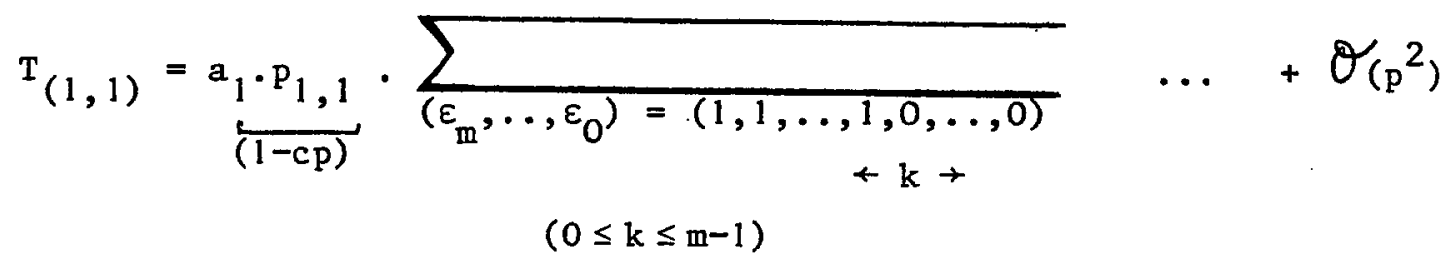$$
=a_{1} \cdot(1-c p) \cdot\left\{\left[\left(1-a_{1}\right)(1-c p)\right]^{m-1} \cdot a_{1}+c p a_{0} \cdot a_{1} \cdot \sum_{k=1}^{m-1}\left[\left(1-a_{0}\right) \cdot(1-p)\right]^{k-1} x\right.
$$$$
\left[\left(1-a_{1}\right) \cdot(1-c p)\right]^{m-1-k}+\theta\left(p^{2}\right)
$$

which reduces to the formula given in section 2 .

By using these values we obtain the following expression for the probability that the distance between consecutive errors is m : 


$$
\begin{aligned}
& \operatorname{Pr}\left(s_{m}, \overline{s_{m-1}}, \ldots, \overline{s_{1}} \mid s_{0}\right) \\
& =\frac{1}{1+\frac{a_{1}}{a_{0} c}} \cdot\left(a_{0} \cdot(1-p)+\frac{2 p a_{1}}{1-\beta}\right) \cdot\left[\left(1-a_{0}\right)(1-p)\right]^{m-1} . \\
& \left.\quad+\left(\frac{a_{1}^{2}}{a_{0} c} \cdot(1-c p)+\frac{2 p a_{1}}{1-\frac{1}{\beta}}\right) \cdot\left[\left(1-a_{1}\right)(1-c p)\right]^{m-1}+\theta_{(p}{ }^{2}\right) .
\end{aligned}
$$

This formula is exact if $\mathrm{p}=0$ or if $\mathrm{m}=1$; it has been used to evaluate the average distance between consecutive errors.

The calculation of $T^{\prime}(1,1)$ is similar to the calculation of $T_{(1,1)}$. We obtain :

$$
\begin{aligned}
& T_{(1,1)}^{\prime}=a_{1} \cdot p_{1,1} \sum_{\left(\varepsilon_{m}, \ldots, \varepsilon_{0}\right)=(1,1, \ldots, 1,0, \ldots, 0)} \prod_{i=1}^{m-1}\left(1-a_{\varepsilon_{i}}\right) \cdot p_{\varepsilon_{i-1}, \varepsilon_{i}}+\theta_{\left(p^{2}\right)} \\
& +k \rightarrow \\
& (1 \leq k \leq m-1) \\
& =a_{1} \cdot(1-c p) \cdot \sum_{k=1}^{m-1}\left[\left(1-a_{0}\right) p_{0,0}\right]^{k-1} \cdot\left(1-a_{1}\right) \cdot p_{0,1} \cdot\left[\left(1-a_{1}\right) p_{11}\right]^{m-1-k}+O\left(p^{2}\right) \\
& =a_{1} \cdot p \cdot\left[\left(1-a_{1}\right)(1-c p)\right]^{m-1} \cdot \frac{1-\left(\frac{1}{\beta}\right)^{m-1}}{1-\frac{1}{\beta}}+\mathcal{O}_{\left(p^{2}\right)} \text {. }
\end{aligned}
$$


REFERENCES

[1] E. Gelenbe, C. Poulain: Performance of Protocols in the Satellite Channe1. First International Conference on Distributed Systems, Huntsville 1979, 177 - 184 .

[2] L. Kleinrock: Queueing Systems. Wiley 1976.

[3] J. Labetoulle, G. Pujolle: Modeling and Performance Evaluation of the HDLC Protocol. International Symposium on Flow Control in Computer Networks, Versailles 1979, $307-320$.

[4] J. Martin: Communications Satellite Systems. Prentice-Hal1 1978.

[5] H. Perros, G. Pujolle: On Satellite Communication Protocols. To be presented at Compcon 1980, Washington.

[6] CCITT Recommendation X.25, Orange Book, Vo1. 8, Geneva 1977.

[7] ISO/DIS 4335: High Level Data Link Control Procedures, 1977.

[8.] IBM, COMSAT, French PTT: Computer Satellite Communications Experiments, Phase - Phase 2.

[9] Workshop "Rommunikation in verteilten Systemen", Berlin 1979. Informatik-Fachberichte, Springer 1979. 
